\title{
A Brief Survey on V-Blast Detection in MIMO OFDM System
}

\author{
Pranav Pandey \\ M-Tech Research Scholar \\ Department of Electronics and \\ Communication Engineering \\ TIT, Bhopal
}

\author{
Shivendra Singh \\ Research Guide, \\ Department of Electronics and \\ Communication Engineering \\ TIT, Bhopal
}

\begin{abstract}
Multiple input multiple outputs-orthogonal frequency division multiplexing (MIMO-OFDM) is the key procedure for era correspondences frameworks. In this paper, a few identification calculations dependent upon MIMO-OFDM frameworks are quickly presented. Different input-multipleyield frameworks give an extremely swearing up and down to means to build the ghastly proficiency for remote frameworks. By utilizing orthogonal recurrence division multiplexing (OFDM), wideband transmission might be attained over recurrence particular blurring radio channels. Initially, in this paper, we present an improved vertical Bell Labs layered space-time (V-BLAST) recipient which considers the choice mistakes and second, give short evaluation of diverse calculation.
\end{abstract}

\section{Keywords}

Multiple-input-multiple-output (MIMO) systems, Orthogonal frequency-division multiplexing (OFDM), vertical Bell Labs layered space-time (V-BLAST).

\section{INTRODUCTION}

As Fourth-Generation (4G) remote frameworks are constantly intended for offering excellent interactive media serindecencies, which incorporate voice, information, and feature, the obliged information rates will expand considerably contrasted and existing administrations. So as to fulfill this developing interest, extensive exploration considerations have been concentrated on enhancing the otherworldly effectiveness in remote channels. Arriving at this objective requires a proficient utilization of the constrained remote data transfer capacity asset. Ordinarily, remote channels show various serious disabilities, for example, intersymbol obstruction (ISI) and blurring, which prompt extraordinary tests in cutting edge remote correspondences. As higher bit rates lead to wideband interchanges, wire- less channels gets to be recurrence specific. Multicarrier regulation acknowledged by orthogonal recurrence division multiplexing (OFDM) is appropriate for such broadband provisions [1], [2].

The OFDM tweak strategy separates the aggregate accessible data transfer capacity into various just as dispersed recurrence groups. By applying a fitting cyclic prefix, singular sub channels are changed to show flat blurring channel qualities. The layered

Space-time building design recommended in [3] has guaranteed greatly high ghostly proficiency in various inputnumerous yield (MIMO) frameworks. Around such spatialdivision multiplexing (SDM) methods, vertical Bell Labs layered space-time (V-BLAST) [4] displays the best tradeoff between execution and unpredictability. The V-BLAST utilizes a consolidation of straight and nonlinear identification systems: first nulling out the impedance from yet undetected indicators, and after that counteracting the obstruction utilizing officially recognized signs [4].

Nonetheless, the conventional systems for image identification embraced in the V-BLAST don't work well when the channel coding is connected, since the decoder execution generously experiences the mistake proliferation natural in the choice criticism process. Consequently, the collector needs to make up for the slip engendering preceding the channel decoder. The quick expanding interest for high velocity remote information transmission has postured incredible tests for remote framework designation to acknowledge higher throughput remote correspondences in radio channels with constrained transfer speed. Generally talking, basic figures of legitimacy for a correspondence framework incorporate high information throughput to its terminals and low bit/outline failure rates. Lately, various transmit and get reception apparatuses are less averse to be viewed as a solid result in future broadband remote correspondence frameworks, as the limit of such a different info numerous yield (MIMO) channel expands straightly with the augmentation of the base between the amount of transmit and get recieving wires in a rich- the earth, without expanding the transmission capacity or transmitted force [3]

As a streamlined adaptation of the spatial multiplexing method, the Vertical Bell Laboratories Layered Space- Time (V-BLAST) plan can accomplish high unearthly proficiency while upholding generally low usage many-sided quality [4]. Numerous studies have been led to attain better execution or more level usage many-sided quality. Whatever remains of this paper is sorted out as takes after. In Section II, we portray MIMO-OFDM frameworks model. In Section III, clarify past consequence and finally concludes follow in section IV.

\section{MIMO-OFDM SYSTMS MODEL}

When presenting the sign identification, we quickly portray a MIMO-OFDM framework. The consolidation of OFDM and VBLAST can overcome intersymbol impedance in recurrence specific blurring channels. A multicarrier framework might be productively executed in discrete time utilizing a backwards FFT (IFFT) to go about as a modulator and a FFT to go about as a demodulator. The VBLAST building design is dependent upon a solitary transporter indicator transforming calculation. Accordingly, to join together it with OFDM, the VBLAST discovery process must be performed on every subcarrier at the recipient. The definite framework design of the VBLASTOFDM is indicated in figure1-2.

\subsection{MIMO-OFDM}

MIMO-OFDM frameworks Consider a correspondence framework with nth transmitting receiving wires and $\mathrm{nr}$ getting radio wire, indicated by $\left(\mathrm{n}_{\mathrm{t}}, \mathrm{n}_{\mathrm{r}}\right)$. Figure 1 is chart of MIMO-OFDM transmitter. At the transmitter the information 
bit stream is de-multiplexed and coded to create image streams that are propelled into the IFFT modulators and included cyclic prefix (CP). At long last, the OFDM signs are transmitted over every transmit reception apparatus. Figure 2 shows the piece outline of a VBLAST-OFDM collector. Every recipient reception apparatus beneficiaries signs sent from all transmit radio wires. After the cyclic prefix is uprooted, each one gained sign passes through a FFT hinder for demodulation. At the collector, we accept flawless OFDM synchronization. The collector motion after demodulation, at accept reception apparatus $\mathrm{j}$ for sub channel, is given by

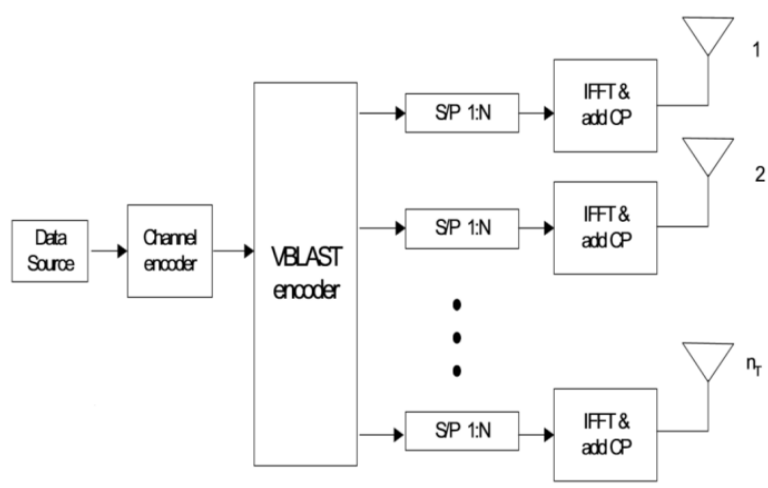

Fig. 1 VBLAST-OFDM transmitter

$$
r_{j}[n, k]=\sum_{i} H_{i j}[n, k] t_{i}[n, k]+w_{j}[n, k] \text { (1) }
$$

Where $i=1,6, n t, j=1,6, n r, t i[n, k]$ is the transmitted image from the ith transmit reception apparatus at $\mathrm{k}$ sub channel, $w j[n, k]$ in (1) indicates the added substance complex Gaussian commotion at the Jth beneficiary radio wire, and is thought to be zero-mean with change on2. $\mathrm{H}_{\mathrm{ij}}$ $[\mathrm{n}, \mathrm{k}]$ in (1) means the channel recurrence reaction for the $\mathrm{k}^{\mathrm{th}}$ tone at time $\mathrm{n}$, comparing to the ith transmit and the beneficiary reception apparatus.

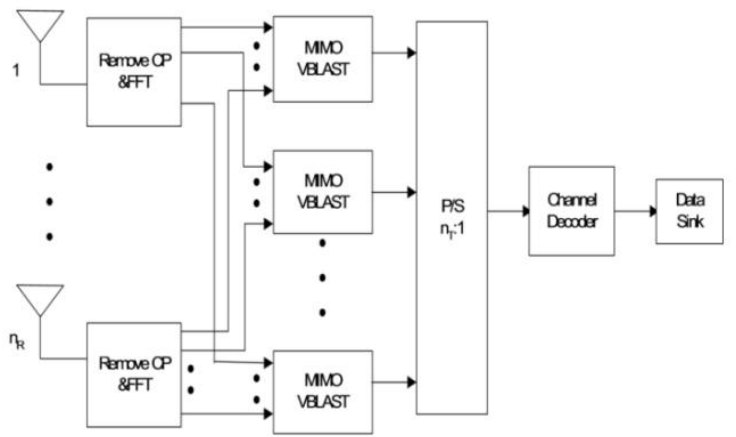

Throughout the gathering, every recipient radio wire gets the sign transmitted from all the transmit receiving wires. To start with, the cyclic prefix of each one gained indicator is uprooted. In the wake of passing through a serial-to-parallel converter and the quick Fourier change pieces (FFTs), the subcarriers are divided. At that point, the $\mathrm{N}$ data images fitting in with every subcarrier are steered to their relating VBLAST multi- radio wire handling unit where the de-mapping and disentangling are performed. The caught bits are changed over go into a serial structure likewise to recoup the transmitted information bits.

\subsection{Channel Models}

We expect that the OFDM sign is transmitted over a remote interchanges environment by a multipath blurring channel and a given lucidness transmission capacity. The complex baseband likeness a blurring channel drive reaction from transmit reception apparatus $i$ to collector receiving wire $j$ could be communicated as,

$$
h i j(t, \tau)=\sum_{l=1}^{L} \alpha_{i j}^{l}(t) \delta\left(\tau-\tau_{1}\right)
$$

Where $\alpha_{\mathrm{ij}}^{1}(\mathrm{t})$ are wide-sense stationary narrowband complex Gaussian processes and are assumed to be independent among different paths transmit antenna $i$ to the receiver antenna $j$.L is the number of multipath. Thus for each receive antenna, channel frequency responses used for the $\mathrm{k}^{\text {th }}$ subcarrier at time $\mathrm{n}$ is

$$
H_{i j}[n, k]=\sum_{l=0}^{L-1} \alpha_{i j}^{1}(t) e^{-j 2 k f \tau_{1}}
$$

Where $\mathrm{f}=\mathrm{B} / \mathrm{K}$ is the subcarrier spacing, $\mathrm{B}$ is the total bandwidth, and $\mathrm{K}$ is the total number of subcarriers.

\section{LITERATURE REVIEW}

Mr. Shreedhar A. Joshi, Dr. Rukmini T. S. Dr. Mahesh H. M. [6], frameworks have as of late developed as a key innovation in remote correspondence frameworks for expanding both information rates and framework execution. The Vertical Bell Labs Space-Time Architecture (V-BLAST) is one of the plans, which could be connected to MIMO remote framework. This paper proposes sign finders for V-BLAST building design with Maximum Likelihood (ML), Zero- Forcing (ZF), Minimum Mean-Square Error (MMSE), and Successive Interference Cancelation (SIC) identifiers and mimics these structures in Rayleigh blurring channel. The proposed dissection additionally contrasts the exhibitions of MIMO framework and distinctive tweak strategies like BPSK and QPSK in Fading and AW GN channels. In light of bit slip rates, we investigate the execution and the computational unpredictability of these plans.

Fig. 2 VBLAST-OFDM receivers

Table 1 Summary of Literature Review

\begin{tabular}{|c|c|c|c|c|}
\hline Year & Author & \multicolumn{2}{c|}{ Title } & Approach \\
\hline \multirow{2}{*}{2012} & $\begin{array}{c}\text { Mr. Shreedhar } \\
\text { A. Joshi, Dr. } \\
\text { Rukmini T. S. } \\
\text { Dr. Mahesh H. } \\
\text { M. }\end{array}$ & $\begin{array}{c}\text { Error Rate Analysis of the } \\
\text { V-BLAST MIMO Channels } \\
\text { using Interference } \\
\text { Cancellation Detectors }\end{array}$ & $\begin{array}{c}\text { Signal detectors for V-BLAST } \\
\text { architecture with Maximum } \\
\text { Likelihood (ML), Zero- Forcing (ZF), } \\
\text { Minimum Mean-Square Error } \\
\text { (MMSE), and Successive Interference } \\
\text { Cancellation (SIC) detectors }\end{array}$ & $\begin{array}{c}\text { Increasing both data } \\
\text { rates and system } \\
\text { performance }\end{array}$ \\
\hline \multirow{2}{*}{2010} & $\begin{array}{c}\text { Shreedhar. A. } \\
\text { Joshi, Dr. Rukmini } \\
\text { TS, Dr.Mahesh H } \\
\text { M, }\end{array}$ & $\begin{array}{c}\text { Performance Analysis of } \\
\text { MIMO Technology using V- } \\
\text { BLAST Technique for } \\
\text { Different Linear Detectors }\end{array}$ & $\begin{array}{c}\text { Zero forcing (ZF), Minimum Mean } \\
\text { Square Error (MMSE) with back } \\
\text { substitution SIC }\end{array}$ & $\begin{array}{c}\text { V-BLAST achieves } \\
\text { better Bit error rates }\end{array}$ \\
\hline
\end{tabular}




\begin{tabular}{|c|c|c|c|c|}
\hline & & in a Slow Fading Channel & & \\
\hline 2009 & $\begin{array}{l}\text { Kai Wu, Lin } \\
\text { Sang, He Wang, } \\
\text { Cong Xiong, } \\
\text { Dacheng Yang, } \\
\text { Xin Zhang, }\end{array}$ & $\begin{array}{l}\text { Detection Algorithm for V- } \\
\text { BLAST Systems with Novel } \\
\text { Interference Cancellation } \\
\text { Technique }\end{array}$ & $\begin{array}{l}\text { Zero-forcing ordered successive } \\
\text { interference cancellation (ZF-OSIC) or } \\
\text { minimum mean square error ordered } \\
\text { successive interference cancellation } \\
\text { (MMSE-OSIC) detection algorithm }\end{array}$ & $\begin{array}{l}\text { More feasible for } \\
\text { practical applications }\end{array}$ \\
\hline 2008 & $\begin{array}{l}\text { Wu Nian Wang } \\
\text { Zhongpeng } \\
\text { Zhang } \\
\text { Shaozhong, }\end{array}$ & $\begin{array}{c}\text { Performance Comparison } \\
\text { of Sub-optimal Algorithms } \\
\text { Based on MIMO-OFDM } \\
\text { Systems }\end{array}$ & $\begin{array}{c}\text { Several detection algorithms based on } \\
\text { MIMO-OFDM systems }\end{array}$ & $\begin{array}{l}\text { ZF-PIC algorithm is } \\
\text { very attractive } \\
\text { compared to } \\
\text { conventional ZF- } \\
\text { VBLAST in } \\
\text { performance } \\
\end{array}$ \\
\hline 2007 & $\begin{array}{l}\text { Jiming Chen, } \\
\text { Shan Jin, and } \\
\text { Yonggang } \\
\text { Wang, }\end{array}$ & $\begin{array}{l}\text { Reduced Complexity } \\
\text { Mmse-Sic Detector In V- } \\
\text { Blast Systems }\end{array}$ & $\begin{array}{l}\text { Conventional successive interference } \\
\text { cancellation (SIC) detection algorithm }\end{array}$ & $\begin{array}{l}\text { Computational } \\
\text { complexity of the } \\
\text { proposed detection } \\
\text { algorithm is } \\
\text { significantly reduced }\end{array}$ \\
\hline
\end{tabular}

Shreedhar. A. Joshi, Dr. Rukmini T S, Dr.Mahesh H M [7], The V-BLAST (Vertically - layered Bell Laboratories Layered Space-Time) calculation has the multi- layer image location plan. The proposed work concentrates on V-BLAST method with a Multiple Input Multiple yield (MIMO) innovation took after by collector discovery strategies like Zero driving (ZF), Minimum Mean Square Error (MMSE) with the back substitution SIC (Symbol Interference Cancelation). The proposed approach receives BPSK, QAM balance techniques. The numerical examination is led utilizing MATLAB. The execution change is additionally noteworthy and a Simulation effect indicates that V-BLAST accomplishes better Bit error rates.

Kai Wu, Lin Sang, He Wang, Cong Xiong, Dacheng Yang, Xin Zhang [8], In the tried and true zero-compelling requested progressive obstruction scratch-off (ZF-OSIC) or least mean square failure requested progressive impedance retraction (MMSE-OSIC) discovery calculation of vertical Bell-Labs layered space-time (V- BLAST) building design, there exists an undesirable preparing defer because of the count framework pseudo-converse and force reordering in identifying the image in each one layer. The parallel impedance dropping (PIC) calculation has an easier handling postpone yet poorer execution. In light of the characteristics of SIC and PIC calculations, an incorporated PIC and OSIC discovery (IPOD) calculation is proposed which can bring down the unpredictability and also the preparing postponement of the entire calculation with little execution misfortune. Also, there is an alterable parameter in IPOD calculation which could be changed to acquire distinctive tradeoff between the multifaceted nature and execution. In this means, the proposed calculation is more doable for commonsense provisions

Wu Nian Wang Zhongpeng Zhang Shaozhong [9], is the key procedure for era interchanges frameworks. In this paper, a few location calculations dependent upon MIMO-OFDM frameworks are quickly presented, and their exhibitions are assessed by machine reproduction. The reproduction effects demonstrate that execution of these calculations for MIMOOFDM framework is like the execution for even MIMO framework. We infer that the so- called ZF-PIC calculation is exceptionally alluring contrasted with accepted ZF-VBLAST in execution.

Jiming Chen, Shan Jin, and Yonggang Wang [10], studies have indicated that layered space-time architectures like the Vertical Bell labs Layered Space-Time (V-BLAST) framework can misuse the limit preference of various reception apparatus frameworks in rich-disseminating situations. In this paper, we display a decreased unpredictability calculation for recognizing such structural engineering regarding the minimum mean square error (MMSE) rule. Calculation bases on the routine progressive impedance scratch-off (SIC) discovery calculation, yet chooses a few layers with sufficiently substantial sign to obstruction in addition to commotion degree (SINRs) rather than the layer with biggest SINR at each one phase of progressive dropping, and uses the Gram- Schmitt Orthogonalization (GSO) for substituting the processing of pseudo-backwards in discovering the weight vectors. Accordingly the computational intricacy of the proposed identification calculation is fundamentally lessened yet the execution corruption is little.

\section{CONCLUSION}

In this paper, a few sub-ideal MIMO beneficiaries are acquainted and they are connected with MIMO-OFDM frameworks. The exhibitions of these calculations are assessed by workstation reenactment in writing table. Recreation states that the exhibitions of these calculations in MIMO-OFDM frameworks are like their exhibitions in MIMO frameworks. The proposed writing work gives the execution assessment of V-BLAST with a few indicators (ZF, MMSE) in moderate blurring channels. Besides, the presentation of SIC plans still enhances the free coded V-BLAST framework. The outcomes of these plans are analyzed in the proposed writing work. Thusly, it is demonstrated that, MIMO is an imperative key engineering for the Fourth era remote systems. 


\section{REFERENCES}

[1] L. J. Cimini, "Analysis and simulation of a digital mobile channel using orthogonal frequency division multiplexing," IEEE Trans. Commun., vol. 33, no. 7, pp. 665-675, Jul. 1985.

[2] J. A. Bingham, "Multicarrier modulation for data transmission: An idea whose time has come," IEEE Commun. Mag., vol. 28, no. 5, pp. 5-14, May 1990.

[3] G. J. Foschini, "Layered space-time architecture for wireless commu- nication in a fading environment when using multi-element antennas," Bell Labs. Tech. J., vol. 1, pp. 41-59, 1996.

[4] P. W. Wolniansky, G. J. Foschini, G. D. Golden, and R. A. Valenzuela, "V-BLAST: An architecture for realizing very high data rates over the richscatteringwirelesschannel,"inProc.URSIInt.Symp.Signals , Syst., Electron. Sep. 1998, pp. 295-300.

[5] G.J. Foschini, M. J. Gans, "On limits of wireless communications in a fading environment when using muptiple antennas," Wireless Personal Comm., vol. 6, pp. 311-335, Mar. 1998.

[6] Mr. Shreedhar A. Joshi, Dr. Rukmini T. S. Dr. Mahesh H. M., "Error Rate Analysis of the V-BLAST MIMO
Channels using Interference Cancellation Detectors" 2012 IEEE.

[7] Shreedhar. A. Joshi, Dr. Rukmini T S, Dr.Mahesh H M, "Performance Analysis of MIMO Technology using VBLAST Technique for Different Linear Detectors in a Slow Fading Channel" 2010 IEEE.

[8] Kai Wu, Lin Sang, He Wang, Cong Xiong, Dacheng Yang, Xin Zhang, "Detection Algorithm for V-BLAST Systems with Novel Interference Cancellation Technique" 2009 IEEE Wireless Theories and Technologies Lab (WT\&T) Beijing University of Posts and Telecommunications, Beijing, P.R.China.

[9] Wu Nian Wang Zhongpeng Zhang Shaozhong, "Performance Comparison of Sub-optimal Algorithms Based on MIMO-OFDM Systems" $11^{\text {th }}$ IEEE international conference on communication technology proceedings 2008.

[10] Jiming Chen, Shan Jin, and Yonggang Wang, "Reduced Complexity MMSE-SIC Detector in V-Blast Systems" The 18th Annual IEEE International Symposium on Personal, Indoor and Mobile Radio Communications (PIMRC'07) 2007. 